\title{
Cadres de participation en transition: une perspective multimodale sur l'organisation de la parole en classe
}

\author{
Virginie Fasel Lauzon \& Fee Steinbach Kohler \\ Université de Neuchâtel (Suisse) \\ virginie.fasel@unine.ch \\ Université de Bâle (Suisse) \\ fee.steinbach@unibas.ch \\ Evelyne Berger \\ Université de Neuchâtel (Suisse) \\ evelyne.berger@unine.ch
}

\section{Introduction}

Dans la classe de français langue première prennent place des activités multiples et variées dont le but pédagogique commun est de développer la compétence d'interaction des élèves tout en travaillant à la construction de connaissances disciplinaires (notamment liées, au niveau du secondaire supérieur, à l'analyse de textes littéraires). Interagir en classe demande aux participants de gérer de nombreux aspects liés d'une part au cadrage institutionnel, qui instaure a priori une asymétrie des rôles (enseignant $v s$. élève), et de l'autre à la présence d'une multitude de co-participants, tous étant des divers participants quant à la sélection du prochain locuteur ou à l'identification du destinataire. L'organisation de la prise de parole en classe est ainsi particulièrement complexe. On observe donc que la majorité des échanges se déroulent sous forme d'une structure tripartite initiation-réaction-follow-up $(I R F)$, laquelle est gérée de manière centrale par l'enseignant. Cependant, une analyse détaillée d'interactions en classe permet de dégager certains épisodes interactionnels qui ne peuvent être décrits sous la forme de structures IRF. Ces épisodes se distinguent par une participation active de plusieurs élèves qui s'adressent directement les uns aux autres, exploitant ainsi le potentiel lié à la présence de nombreux co-participants. Il en résulte une grande richesse discursive et interactionnelle qui s'observe notamment dans la succession ou la coexistence de plusieurs cadres de participation gérés collaborativement.

Le présent article se propose d'adopter une approche micro-analytique basée sur des enregistrements vidéo pour étudier ces différents cadres de participation et de dégager la complexité de leur dynamique multimodale, en faisant émerger la manière dont des discours appartenant à différentes sphères de légitimation émergent, s'influencent et se croisent (cf. Mondada, 2003). Il prend plus spécifiquement pour objet central les moments d'émergence et de transition entre cadres de participation pour montrer, au moyen d'une analyse prenant en compte aussi bien les éléments proprement linguistiques que les gestes, regards et orientations corporelles, comment ces moments sont négociés et construits localement par les participants de manière collaborative. Ces analyses permettront à leur tour de réfléchir au potentiel de ces moments de transition tant pour la co-construction des contenus disciplinaires que pour la mise en œuvre et la pratique de ressources complexes sur le plan interactionnel.

\section{Dynamique des prises de parole en classe}

La classe en tant que lieu institutionnel repose sur une attribution de droits et d'obligations des participants en fonction des rôles envers lesquels ils s'orientent (cf. les études sur les interactions en milieu institutionnel, notamment Heritage \& Greatbach, 1991). A cet aspect vient s'ajouter la caractéristique du nombre élevé de participants, et donc de locuteurs et interlocuteurs potentiels, d'où la nécessité pédagogique d'une centralisation de l'attention gérée par l'enseignant (van Lier, 1988). Pour cette raison, il est peu étonnant que les études classiques sur les interactions en classe aient relevé la 
présence massive du schéma tripartite initiation-réaction-follow-up (IRF, aussi appelé triadic dialogue), mettant l'accent sur le rôle structurant de l'enseignant quant aux formes de participations adéquates. Ainsi, Sinclair et Coulthard (1975) proposent une typologie des échanges en classe dont le format prototypique est initiation-réaction-feedback. Mehan $(1979 ; 1985)$, s'inspirant des travaux en ethnométhodologie, présente une description en termes analogues de IRE (E pour évaluation), insistant davantage sur le caractère co-construit de ces séquences, décrites comme un emboîtement de deux paires adjacentes (Sacks, Schegloff \& Jefferson, 1974). Bien que la structure tripartite soit le schéma le plus répandu en salle de classe (Wells, 1993; Hall, 2007), il ne reflète pas de manière exhaustive la réalité complexe des échanges en classe dans la mesure où il ne permet pas de rendre compte du caractère processuel, localement négocié et co-construit des interactions.

Ces dernières années, des études empiriques menées dans une perspective inspirée par l'analyse conversationnelle se sont attachées à montrer que la gestion des formats d'activités fait l'objet de négociations et d'ajustements constants entre les interactants sur le plan local (cf. p.ex. Gajo \& Mondada, 2001; Hall, 2004, 2007; Markee, 2004; Mondada \& Pekarek Doehler, 2004; Mortensen, à paraitre; Pekarek, 1999; Pekarek Doehler, 2002; Seedhouse, 2004). Ces travaux déconstruisent une vision des interactions en classe qui seraient régies exclusivement par un système prédéfini. La tâche didactique ne représente qu'un projet (blue-print, cf. Coughlan \& Duff, 1994) transformé dans l'activité localement située et accomplie. Bien que les apprenants soient en général contraints à participer à l'accomplissement d'une tâche pédagogique prédéfinie, une perspective micro-analytique et multimodale permet de mettre en évidence que la tâche fait l'objet d'une gestion locale, accomplie dans la séquentialité des actions des participants (Hall, 2004; Mondada \& Pekarek Doehler, 2004). La classe constitue donc un lieu d'interactions dynamiques complexes (Cicurel, 2002; cf. aussi Hall, 2004 qui parle des interactions en classe comme une constellation de pratiques interactionnelles complexes et intriquées: 608) où l'organisation des activités discursives est configurée par l'ensemble des participants. Cette organisation n'est pas imposée par un modèle préexistant et statique mais accomplie localement; elle est donc modifiable à chaque moment de l'interaction. Dès lors, il existe une possibilité réelle pour l'élève de participer à la gestion et à la co-construction de l'interaction sur le plan de l'organisation des échanges et notamment la prise de parole.

Les possibilités pour les élèves d'obtenir ou de prendre la parole en classe reposent sur différentes modalités d'attribution du tour: 1) hétéro-sélection d'un élève particulier par l'enseignant; 2) hétérosélection de l'ensemble de la classe (comme collectif) par l'enseignant (Lerner, 1993) qui donne lieu soit à une action collective (p.ex.: réponse en chœur), soit à une action individuelle, 3) hétéro-sélection par sollicitation (le plus souvent en levant la main) et 4) auto-sélection. Dans ce dernier cas de figure, l'élève se trouve placé devant plusieurs problèmes pratiques: exhiber sa position de locuteur imminent (incipient speaker) et établir un rapport interlocutif avec un destinataire choisi (recipiency) afin d'établir un cadre de participation au sein duquel puisse émerger le discours (cf. Mortensen, à paraître). Par ailleurs, le nombre élevé de participants offre la possibilité d'une multitude d'échanges croisés, notamment en parallèle de l'interaction officielle. Les cas d'auto-sélections apparaissent dans des épisodes interactionnels ne pouvant être réduits au schéma prototypique IRF, contrairement aux trois autres modalités (ci-dessus). Ces cas demandent pour être analysés de faire appel à des outils méthodologiques et à un cadrage épistémologique permettant de suivre pas-à-pas le déroulement interactionnel dans toute sa complexité. Pour cette raison, nos analyses s'inscrivent dans la lignée conversationnaliste et se proposent de prendre en considération la dimension multimodale - éléments encore trop peu exploités pour l'analyse des interactions en classe (mais cf. Mortensen, à paraître) - notamment les regards et les orientations corporelles, pour documenter l'établissement et la transition des configurations interactionnelles que nous capturons par la notion de cadre de participation.

\section{Cadrage épistémologique et méthodologique}

Afin de comprendre la manière subtile dont les acteurs gèrent les interactions en classe au-delà des structures IRF, nos analyses sont menées dans l'esprit de l'Analyse Conversationnelle d'inspiration ethnométhodologique (cf. Sacks, 1992, Sacks, Schegloff \& Jefferson, 1974; Schegloff, 1988, 1995 
Schegloff \& Sacks, 1973 pour des études classiques et Gülich \& Mondada, 2001 pour une introduction en français). Nous exploitons notamment la notion de participation en tant que concept analytique (cf. Goffman, 1981 et Goodwin \& Goodwin, 2004) pour rendre visible ce qui se passe au niveau local des échanges complexes en classe de langue ${ }^{1}$.

La notion de participation a initialement été forgée par Goffman (1981) pour qui l'interaction ouvre « tout un éventail de possibilités structurellement différenciées » qui établit le cadre «au sein duquel le locuteur dirige sa production » (Goffman, 1981: 147). Par la suite, cette notion a été élaborée de façon critique notamment par les Goodwins (Goodwin \& Goodwin, 2004; Goodwin, 2007b) qui l'appliquent à l'analyse d'instances interactionnelles concrètes, et c'est dans cette filiation que nous inscrivons nos analyses (pt. 5) ${ }^{2}$. La critique adressée à la conception originale goffmanienne porte essentiellement sur un manque de dynamisme dans la typologie proposée par Goffman et sur le fait que les dimensions locuteur(s) et auditeur(s) ne sont analytiquement pas traitées de façon différente et à l'aide de concepts différents (Goodwin \& Goodwin, 2004). Outre cette critique, Goodwin \& Goodwin (2004) proposent une re-conceptualisation de la notion de participation en se focalisant sur les pratiques et la participation comme action incarnée. Ainsi, ils mettent davantage l'accent sur la nature dialogique et intrinsèquement réflexive qui lie les interlocuteurs dans un cadre de participation commun et complexe. Le cadre de participation correspond à l'ensemble des statuts de participation de tous les interactants dans une situation donnée, à un moment donné (Goodwin \& Goodwin, 2004). Il s'agit dès lors de décrire les pratiques qui émergent autant dans l'établissement de cadres que dans leur transformation dynamique et continuelle au cours d'une interaction. Or, en se focalisant sur la dimension linguistique uniquement, bon nombre de phénomènes pertinents pour la participation ne sont plus disponibles pour l'analyse (Mondada, 2003). Ainsi, à côté de la structure linguistique des énoncés, la prosodie, le rythme, les regards, les orientations corporelles et la manipulation d'objet sont des ressources indispensables à prendre en compte puisqu'elles permettent aux interactants de projeter, d'interpréter et d'organiser leurs contributions et de co-construire leurs interactions. La critique des approches verbocentrées, présente déjà chez Goffman qui ouvre sa réflexion à l'importance des dimensions non-verbales, notamment aux regards et aux orientations corporelles (Goffman, 1981), est prolongée de façon conséquente dans la reconceptualisation interactionniste qui prend en compte l'ensemble des dimensions multimodales, le verbal aussi bien que le non-verbal. Dans une perspective praxéologique, émanant de l'analyse conversationnelle, le point de départ analytique n'est pas la parole, le discours du seul locuteur, mais l'activité, les pratiques incarnées de l'ensemble des participants. La participation est logée dans des activités situées, et l'approche multimodale nous permet d'observer « how both speakers and hearers as fully embodied actors and the detailed organization of the talk in progress are integrated into a common course of action ${ }^{3} \gg$. (Goodwin \& Goodwin, 2004: 226). Schegloff (1988) par exemple montre comment le tour complexe d'un locuteur est en fait façonné pas-à-pas par la réaction incarnée, non-verbale, de son interlocuteur et relève ainsi d'un accomplissement interactif des deux participants. En tant que concept analytique, la notion de participation fournit dès lors un cadre pour analyser comment les participants s'engagent dans une action, tout en se manifestant mutuellement cet engagement et leur interprétation de l'action (Goodwin \& Goodwin, 2004: 240).

L'analyse conversationnelle représente un outil méthodologique et un cadrage épistémologique pertinens pour suivre la formation et la transition de cadres dans les échanges en classe. Sa perspective microanalytique se focalise sur la dimension localement accomplie des interactions dans la séquentialité des enchaînements des tours de paroles ainsi que sur les éléments d'ordre multimodal (cf. au sujet de la multimodalité notamment les travaux de Goodwin, 1979; Goodwin, 2000; Goodwin, 2007a). L'échange repose sur un accomplissement pas-à-pas du discours, qui demande aux participants de rendre reconnaissable ce qu'ils sont en train de faire, la manière dont ils interprètent le contexte et leurs attentes. Par conséquent, il ne s'agit pas uniquement de décrire l'organisation des activités mais de montrer comment cette organisation s'ancre dans les orientations des participants:

«In particular, this approach means focusing on how interactional rules and practices are ceaselessly drawn on by the participants in constructing shared and specific understandings of where they are within a social interaction. Central to this process is a reflexive dimension in social action: By their actions participants exhibit an analysis or an understanding of the event in which they are engaged, 
Dans cette perspective, chaque détail, qu'il soit de nature linguistique, prosodique, gestuelle ou autre, prend son sens dans le contexte séquentiel où il apparaît. Dès lors, une telle approche implique de travailler sur un corpus d'interactions authentiques, rendues accessibles par leur enregistrement. Les enregistrements vidéo permettent, sinon de préserver la totalité de l'échange, du moins de rendre disponible un certain nombre de détails interactifs vers lesquels s'orientent les interactants eux-mêmes, et de reconstituer les procédés des participants dans une perspective émique (cf. Mondada, 2003). Pour cette raison, nos analyses se basent sur des interactions en classe de langue, recueillies au moyen d'enregistrements audio et vidéo et transcrites dans leurs détails multimodaux. Dans nos analyses, nous nous focalisons sur les cadres de participation comme accomplissement situé indispensable pour l'émergence du discours (cf. à ce sujet Mortensen, à paraître) tout en le façonnant en même temps. Plus particulièrement nous nous intéressons à l'établissement, le changement et la superposition de cadres de participation - ce que nous appelons des cadres en transition.

\section{Corpus et données}

Le corpus sur lequel se basent nos analyses est constitué de leçons de français langue première au niveau de la dernière année de lycée et comprend 12 périodes de 45 minutes. Ces leçons ont été recueillies entre le printemps 2006 et le printemps 2007. Elles ont intégralement fait l'objet d'un enregistrement audio et vidéo ainsi que de transcriptions détaillées selon des conventions inspirées de celles en vigueur en analyse conversationnelle. Les extraits analysés ici ont également fait l'objet d'une transcription multimodale, ayant principalement pour objectif d'indiquer les orientations corporelles (notamment les regards) des élèves prenant la parole au cours de l'extrait. ${ }^{5}$ Les conventions de transcription sont disponibles en annexe.

Toutes les leçons enregistrées sont constituées d'activités réalisées à l'oral (ce qui n'exclut pas un support écrit). La majorité d'entre elles sont des résumés et des analyses de textes littéraires en préparation de l'examen de maturité (l'équivalent du baccalauréat français). D'autres leçons sont des débats ou des discussions ouvertes sur des pièces de théâtre auxquelles les élèves ont assisté ou participé, sur leur perception de l'école et de l'apprentissage, ou encore sur le passage à l'âge adulte.

\section{Analyses}

\subsection{L'organisation de la participation en classe}

La première étape de notre analyse a consisté à repérer dans notre corpus, au sein des activités en classe entière (par opposition aux travaux de groupe, que nous n'avons pas pris en compte), les différentes configurations interactionnelles afin d'en dégager les caractéristiques sur le plan des structures de participation des élèves. Les différentes configurations recensées sont les suivantes:

1) Dans la configuration interactionnelle la plus fréquente, l'enseignant s'adresse aux élèves, de manière individuelle ou collective. Ses tours de parole sont longs et se terminent tendanciellement par des questions. Les élèves qui interviennent sont hétéro-sélectionnés par l'enseignant et s'orientent physiquement vers lui comme destinataire de leurs tours de parole, qui sont en général courts et se terminent typiquement par des intonations montantes interrogatives (Dabène, 1984), se présentant ainsi comme soumis à l'évaluation de l'enseignant. Cette configuration interactionnelle donne donc lieu en général à une alternance stricte entre tours de parole de l'enseignant et d'un élève, organisés en structure tripartite IRE. Les élèves ne participant pas directement au dialogue prennent alors le rôle d'auditeurs plutôt que de récepteurs de la parole et gardent une position relativement statique, ne 'suivant' que rarement la circulation de la parole du regard. Dans certains cas, la question de l'enseignant peut donner lieu à des réponses chorales. L'enseignant peut 
également instaurer un tour de table lors duquel les élèves prennent la parole les uns à la suite des autres, s'adressant tous à l'enseignant, qui ne fournit son évaluation qu'à la fin de l'activité.

2) La configuration interactionnelle est différente lorsque l'enseignant s'adresse également aux élèves, de manière individuelle ou collective, mais que les élèves qui interviennent, s'ils restent physiquement orientés vers l'enseignant, semblent s'adresser indirectement à un autre élève (ou un groupe d'élèves). Cette orientation indirecte vers un pair par l'entremise de l'enseignant s'observe notamment par des procédés de reprises lexicales ou de structures syntaxiques et de continuité thématique. Dans cette configuration, la structure IRF est en général abandonnée et l'enseignant intervient peu, se contentant souvent du rôle de distributeur de la parole aux élèves qui lèvent la main. Les tours des élèves sont alors de taille variable, pouvant être significativement plus longs que dans la première configuration (Pekarek, 1999).

3) Dans certaines situations, plus rares, les élèves s'orientent physiquement et verbalement les uns envers les autres, souvent en employant des termes d'adresse directs (comme le pronom 'tu'). L'enseignant n'intervient alors pas ou peu et les élèves s'auto-sélectionnent de manière spontanée. Cette troisième configuration semble émerger de manière privilégiée dans des situations de désaccord entre élève, ou de construction du sens en collaboration (Fasel Lauzon \& Berger, 2008; Gajo \& Mondada, 2001).

Il est important de noter que nous ne considérons pas ces trois configurations interactionnelles comme des 'types' distincts et mutuellement exclusifs, mais plutôt comme des réalisations possibles à placer sur un continuum, aux extrémités duquel on trouverait d'une part un dialogue enseignant-élève(s) géré sur le plan des contenus et des modalités de prises de parole par l'enseignant uniquement, et d'autre part un dialogue entre élèves géré par les élèves eux-mêmes. Dans les analyses qui suivent, nous nous intéressons particulièrement à la manière dont la transition incarnée entre cadres de participation entraîne des reconfigurations de l'interaction. Ceci ne signifie pas que les cadres se substituent nécessairement les uns aux autres: il peut y avoir coexistence de cadres officiels ou semi-officiels. Un exemple en est les discussions entre élèves voisins de table, qui se caractérisent par un faible volume sonore, mais peuvent acquérir un statut d'officialité en étant par la suite thématisées et intégrées à l'interaction principale (cf. également Fasel Lauzon \& Berger, 2008, pour une autre analyse de la coexistence de plusieurs cadres au secondaire inférieur).

\subsection{Cadre de participation en transition: l'émergence d'un désaccord}

Dans ce premier extrait, on assiste à l'émergence d'un désaccord entre élèves qui conduit à un changement cadre de participation.

\section{Souci de boire (CODI L1-secII-EO-4)}

Situation initiale: les élèves ont sous les yeux le texte du poème "Larme" de Rimbaud; Aishé, Benoît, Camille et Catherine regardent leur texte

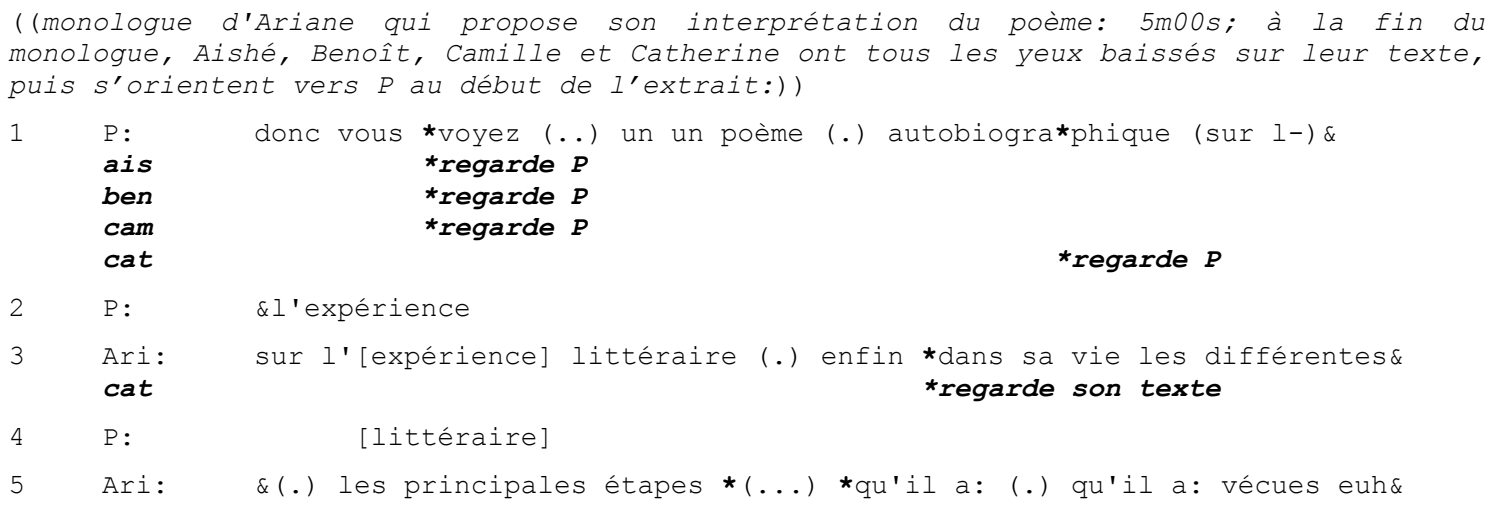


cat

Cat : cam cat

* regarde Catherine

*regarde son texte

\&(.) au niveau de=so:n évolution littéraire

>ouais<

*mais [euhm:] la *dernière *phrase *surtout faut\& [>qu'en pensez vous?<]

*regarde $P$

*regarde $P$

\section{*regarde son texte}

\section{*regarde son texte}

\&*dire (tu l'as fait?)=

*regarde Ariane, la pointe de son pouce gauche

*regarde son texte

=*<ouais> *euh: (...) .h je n'ai pas eu s-je n'ai pas eu souci de\& *regarde son texte

*regarde son texte

\&boire (..) ben (..) c'est sûr que: *[que c'est encore euh:)]

* [ouais ben alors- *ben] ici (..)\&

* regarde rapidement Ariane puis son texte

*pointe une ligne du doigt

\&+*or tel qu'un pêcheur d'or ou de coquillages ((lisant)) +\&

*regarde le texte de Catherine

\&*ben un pêcheur d'or ou: de coquillages *précieux\&

*regarde $P$

*regarde son texte

\&*ben il est (..) très absorbé par son travail (..) genre il doi:t\& * regarde Catherine

\&il doit trier il doit trouver (.) quelque chose *de précieux?\&

* regarde son texte

*regarde Catherine

\&*(..) *et puis il était tellement absorbé par: son but $d-()$.

*regarde son texte

*regarde $P$

* regarde $P$

\&*de devenir voyant (..) *qu'il n'a pas (.) *eu souci de boire\&

*regarde son texte

* regarde son texte

*regarde Catherine

*regarde $P$

\&*C'est-à-dire (..) comme on a v:u au\&

*regarde son texte

*regarde $P$

\&*premier (.) boire ben c'était *la nature euh boire (de-) les\&

* regarde $P$, lève la main puis la baisse et regarde sa feuille

*regarde $P$

\&choses essentielles (..) et ben là il a il a plus (.) été capable

tellement il était absorbé par son travail de penser aux choses

*essentielles.

* lève la main

$\star(1.1)$

* regarde $P$, lève la main

>ben< *moi *je=pense *ça pourrait être *possible mais si on regarde\& *baisse la main 

ais cat cam

cam

*regarde Camille

*regarde son texte *regarde Camille

\section{*regarde son texte}

\&*le: *le (..) le poème il a écrit en mille huit-cents septante-\&

*regarde son texte *regarde son texte

\&deux *>puis en mille huit-cents septante-deux il est\&

*regarde $P$

\&*avec ver*laine il est il a pas encore eu d'échec.\&

*regarde $P$

*regarde Aishé

\&* donc il peut pas être (..) *au courant de *son échec (euh) futur\& *regarde Catherine

*regarde $P$

*regarde $P$

$\& *(\ldots)$ il [est] encore avec $>\star_{\text {verlaine\& }}$

*sourit, regarde sa feuille

*regarde Catherine

[mh ]

\&enfin*[c'est là où il est tout le temps avec verlaine< en\&

*baisse la main, regarde Catherine

\&mille-huit [cent septante-deux]=

$\left[\left({ }^{\circ} \mathrm{C}^{\prime}\right.\right.$ est $\left.\left.(\mathrm{xxxx})^{\circ}\right)\right]$

* [ ${ }^{\circ}$ (votre) théorie $s^{\prime}$ effondre $\left.{ }^{\circ}\right]=$

* geste des deux mains de la bouche vers l'extérieur

$={ }^{\circ}$ non *pas du tout ${ }^{\circ}$ *parce que *il pourrait il il peut très bien\&

*baisse les mains

*regarde Catherine

*regarde Aishé

*regarde son texte

\&*avoir impression (..) ou bien (a) voir déjà l'intuition que (.)\&

* regarde Catherine

*regarde son texte

\&ce=qu'il est en train de fai:re (..) euh: va tomber à l'eau

$\star(\ldots)^{\circ}(\mathrm{x})^{\circ}$

*regarde son texte, prend un feuille dans la main droite

*regarde $P$, lève la main

peut-être *mais moi *il me=semble c'est hyper tôt par rapport à\&

*baisse les yeux

*regarde Camille

\&son *échec qui est quand *même *plus tard\& *regarde Camille

*regarde son texte

*regarde Aishé

\&*(..) après C'e:st c'est ${ }^{\circ}$ à voir ${ }^{\circ}$

*regarde son texte

* regarde Catherine

*hausse les épaules, regarde Camille

ben écoute *[s'il est voyant euh:]

* [c'est possible mais] (..) *>ouais $<() \quad.(\mathrm{xx})=$

* regarde Catherine

* regarde Aishé

*regarde $P$

((rit: 0.8$)$ )

=*mais s'il est voyant ça *veut dire qu'il a pas échoué 


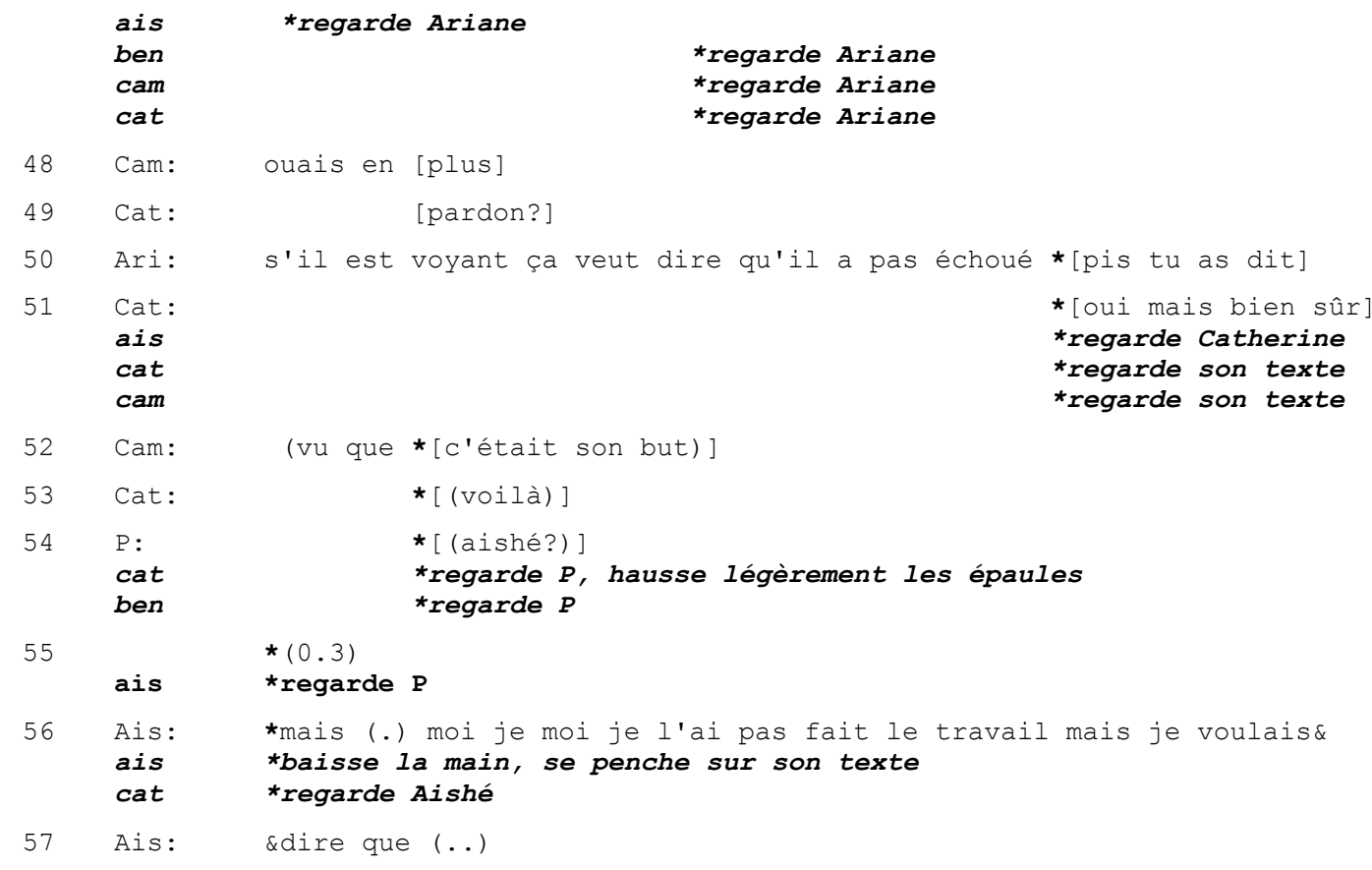

Note: $\mathrm{P}=$ enseignant, Ais $=$ Aishé, Ari $=$ Ariane, $\mathrm{Ben}=$ Benoît, $\mathrm{Cam}=$ Camille, $\mathrm{Cat}=$ Catherine .

Ariane et l'enseignant sont hors-champ de la caméra.

On observe dans cet extrait des orientations 'incarnées' vers plusieurs cadres participatifs en transitions. Les premiers tours (1.1-6) prennent place dans un cadre de participation bien délimité entre l'enseignant et Ariane, qui a pris le rôle de porte-parole de son groupe (dont Catherine fait également partie) et qui vient de présenter son interprétation du poème. Le tour de l'enseignant (1.1-2) prend la forme d'une reformulation à valeur de synthèse de cette interprétation, appelant une confirmation et projetant la clôture de l'exposé du groupe en vue de passer à un autre groupe ou à une autre activité. L'apparente autosélection d'Ariane (1.3) s'explique par le cadre de participation encore en vigueur en moment de préclôture, qui implique comme interlocuteurs principaux l'enseignant et Ariane elle-même en tant que porteparole de son groupe. Ariane confirme en nuançant la proposition de synthèse de l'enseignant en répétant la fin de son tour (sur l'expérience littéraire, 1.3) avant d'introduire une reformulation introduite avec le marqueur enfin (1.3). Avec sa question (1.9), l'enseignant cherche ensuite à ouvrir la conversation au reste de la classe, s'orientant ainsi envers son dialogue avec le groupe comme étant terminé. Or, son tour de parole est chevauché par un tour de Catherine, autre membre du groupe, qui s'auto-sélectionne (1.8). Son intervention, introduite par un mais, poursuit l'activité d'exposé de l'interprétation du groupe. Elle prend place juste après le ouais de l'enseignant suivi d'une pause, qui projette fortement la transition de l'activité. En intervenant à ce moment précis, Catherine contribue ainsi à repousser la clôture du cadre participatif dialogal entre son groupe et l'enseignant. Elle s'adresse d'abord de façon explicite et physiquement incarnée à la porte-parole Ariane, utilisant le pronom d'adresse $t u(1.10)$ et se tournant vers elle et en la pointant du doigt pour solliciter une exemplification de sa part. Suite aux multiples hésitations d'Ariane (1.11-12) qui ne peut accomplir ce que lui demande Catherine, cette dernière reprend à son compte l'activité d'exemplification (1.13-24) orientée vers l'enseignant (dès la 1.14). De par ses orientations corporelles et le moment de son intervention, Catherine maintient une configuration interactionnelle restreinte au sein de laquelle le cadre de participation transite d'une interaction intergroupe à une interaction dirigée vers l'enseignant, remplaçant Ariane dans le rôle de porte-parole. Durant tout son tour de parole (1.13-24), Catherine regarde alternativement l'enseignant et le texte qu'elle a sous les yeux. Cette alternance montre l'importance du texte dans la conversation, qui non seulement constitue le point de référence dans lequel s'ancre le contenu du tour, mais fonctionne également comme une 'voix' à prendre en compte: de la même manière qu'on regarde une personne en train de parler ou dont 
on cite les propos, on regarde le texte pour le lire mais également pour paraphraser ce qu'il 'dit'. Le regard incarne la relation de médiation entre le texte et la discussion qu'il suscite, et justifie ainsi le maintien du cadre de participation centré sur l'interprétation du texte par le groupe dont font partie Ariane et Catherine.

Une transition vers un cadre de participation s'observe aux lignes 24-25. Alors que Catherine termine son tour de parole, deux autres élèves, Aishé et Camille, signalent leur volonté de prendre la parole en levant la main. Le fait qu'aucune de ces deux élèves ne s'auto-sélectionne, contrairement à Catherine, montre qu'elles s'orientent vers un cadre de participation différent. Le début du tour de parole de Camille (1.26), à qui la parole est attribuée par l'enseignant (1.25), enchaîne séquentiellement sur la question que ce dernier avait posée en chevauchement avec le début du tour de Catherine (1.9), bien que sur le plan du contenu propositionnel le tour soit une réaction à l'interprétation du texte d'Ariane et de Catherine. L'orientation de Camille envers le cadre de participation proposé par l'enseignant lors de sa question antérieure est visible de par sa position, tournée vers l'enseignant, le lever de main, ainsi que par la présence du verbe penser en début de tour, qui fait écho à la question de l'enseignant (ben moi je pense, 1.26, cf. qu'en pensez-vous, 1.9). Ces éléments montrent l'orientation des participants envers des normes et pratiques sédimentées en classe tel le rôle structurant de l'enseignant qui attribue la parole; les élèves demandent la parole à l'enseignant même dans des situations où celui-ci semble volontairement se retirer de son rôle dominant, n'évaluant pas la contribution de Catherine (cf. la pause de 1.1 seconde, 1.25) et attribuant la parole de manière non-verbale. Le tour de parole de Camille possède donc une double orientation, vers l'enseignant sur le plan du cadre de participation, et vers ses pairs sur le plan du contenu thématique.

L'interprétation de Camille (1.26-34), remettant en doute celle du groupe constitué par Ariane et Catherine, est orientée vers l'enseignant plutôt que vers Ariane ou Catherine. Cependant, cette interprétation en désaccord fait l'objet d'un alignement manifesté par deux autres élèves, Benoît (1.35) et Aishé (1.36), qui tous deux s'orientent physiquement vers Catherine (Aishé, 1.30; Benoît, 1.31). En marge du tour 'officiel' de Camille s'ébauche donc un cadre de participation 'semi-officiel' qui va constituer le lieu principal de la négociation des interprétations entre les élèves. Juste avant le début du tour de Camille, Aishé s'oriente au départ vers l'enseignant pour solliciter la parole (1.24), mais elle s'était déjà tournée vers Catherine deux fois auparavant (1.5 et 16). Une fois que Camille commence à parler, Catherine, qui est maintenant 'disponible' puisqu'elle a terminé son tour de parole, s'oriente à son tour vers Aishé (1.29), qui croise brièvement son regard avant de se tourner à nouveau vers l'enseignant (1.30). Il semble donc que ce bref regard échangé permette d'établir un terrain pour un échange semi-officiel, qui n'apparait pas étant donné qu'Aishé est en train de lever la main et qu'elle a besoin de maintenir un contact visuel avec l'enseignant pour savoir s'il lui attribue la parole. Le tour de Camille continue mais les multiples reformulations du contenu laisse prévoir une fin prochaine (cf. en 1872 il est avec verlaine, il est encore avec verlaine, enfin c'est là où il est tout le temps avec verlaine en 1872). Pourtant Aishé abandonne alors son projet de prendre la parole de manière officielle, ce qui s'observe dans le fait qu'elle baisse la main et se tourne simultanément à nouveau vers Catherine (1.33). La fin du tour de Camille coïncide alors avec une adresse à mi-voix d'Aishé à Catherine, accompagné d'un geste expressif des deux mains (1.36-37). Catherine s'auto-sélectionne alors de façon immédiate pour exprimer son désaccord d'une manière fortement marquée (absence d'éléments d'atténuation tels que des marques d'hésitations ou d'accord qui retardent le désaccord selon un format préférentiel, cf. Pomerantz, 1984). C'est bien à l'adresse d'Aishé que répond Catherine (1.37), et non à Camille, ce qui s'observe dans la forme du début de son tour: le non pas du tout ne vient pas réfuter le fait que Rimbaud et Verlaine sont ensemble en 1872, mais la conséquence énoncée par Aishé, à savoir que leur interprétation ne résiste pas à ce fait. D'autre part, le début du tour est énoncé à mi-voix, tandis que le reste du tour est énoncé à volume normal: ce faisant, l'interaction entre Aishé et Catherine passe du statut de cadre de participation semi-officiel à officiel et principal, venant remplacer un cadre de participation d'élève à enseignant à un cadre valorisant les échanges directs entre élèves.

Ce nouveau cadre de participation se maintient dans les tours suivants, notamment dans celui de Camille (1.41-43) qui enchaîne de manière contrastive aux propos de Catherine en se tournant vers elle, puis ceux de Catherine (1.44) ou encore Ariane (1.47). Sur le plan linguistique, le passage est caractérisé par un 
registre moins formel, typique des conversations entre élèves (tomber à l'eau, 1.39; c'est hyper tôt, 1.41; ben écoute, 1.44). D'autre part, la dynamique s'accélère comme en rendent compte les nombreux chevauchements $(1.44-45,48-49,50-51,52-53)$ ainsi que les tours de parole plus brefs $(1.44,48,51,52$, 53). Ce cadre de participation ouvert pour gérer le désaccord entre élèves trouve son point culminant à la ligne 47, dans l'intervention d'Ariane. En effet, Ariane faisant partie du groupe de Catherine, le débat est constitué jusqu'à ce moment de deux proposants (Ariane et Catherine) et deux opposants (Aishé et Camille). Or, à la ligne 47, Ariane reprend un argument de Catherine (s'il est voyant, 1.44) pour en modifier l'orientation argumentative (s'il est voyant ça veut dire qu'il a pas échoué, 1.47), s'alignant ainsi à l'argumentation d'Aishé et Camille. Bien que ce tour n'ait pas été énoncé en chevauchement, Catherine lui demande de répéter (1.49), ce qui peut être une demande de confirmation ou une manière de gagner du temps pour faire face au changement de camp inattendu de sa partenaire. Ariane répète alors son argument (1.50) auquel Catherine choisit de s'aligner (1.51). Cet alignement pourrait préparer l'introduction d'une réfutation, que Catherine cherche peut-être dans son texte mais ne semble pas trouver. Suite à une longue pause, elle tourne son regard vers l'enseignant et énonce le mot de clôture voilà (1.53) accompagné d'un haussement d'épaule par lequel elle manifeste sa volonté de clore la discussion et de ne pas continuer d'argumenter seule contre trois en faveur de sa propre interprétation.

En parallèle de ce cadre de participation privilégiant les interactions entre pairs et qui a émergé à l'avantplan de l'interaction, le cadre de participation antérieur, impliquant l'enseignant et les élèves, n'est pas abandonné pour autant par tous les élèves. Il est momentanément en suspend, mais bien présent, ce qui s'observe notamment par le fait qu'Aishé, dès la fin du tour de Catherine lui répondant, s'oriente à nouveau vers l'enseignant pour obtenir la parole, en levant la main (1.40). Durant la suite de l'interaction, elle s'oriente parfois vers ses pairs mais sans intervenir et toujours en gardant la main levée. Aussitôt que Catherine manifeste à l'enseignant sa volonté de clore la séquence entre pairs, l'enseignant donne alors la parole à Aishé, et c'est le cadre participatif initial entre enseignant et élèves qui est rétabli.

Cet extrait se montre d'une grande richesse sur le plan tant verbal que non-verbal. Il permet de suivre pas à pas la transition entre cadres de participation, ainsi que de dégager la manière dont les ressources multimodales sont mobilisées par les participants pour procéder à ce changement. Nous avons vu comment sont entrelacés des discours officiels et non-officiels et comment un cadre de participation peut émerger d'un discours non-officiel, et également que les élèves gèrent activement et efficacement tant l'émergence d'une nouvelle configuration interactionnelle que son déroulement et sa clôture, alors qu'ils sont cependant rarement amenés à le faire dans le contexte scolaire, la gestion de l'interaction étant en général prise en charge par l'enseignant.

\subsection{Cadre de participation en transition: la construction collective d'une explication}

Nous avons dit que l'émergence d'une configuration interactionnelle privilégiant les échanges entre pairs prenait place principalement dans les désaccords et dans les épisodes de construction collective d'un discours. L'exemple ci-dessous, dont nous allons proposer une analyse, montre justement une séquence d'explication collective élaborée entre pairs. Il provient de la même classe que l'exemple précédent, mais d'une autre leçon, qui quoiqu'également axée sur Rimbaud, porte sur deux autres poèmes.

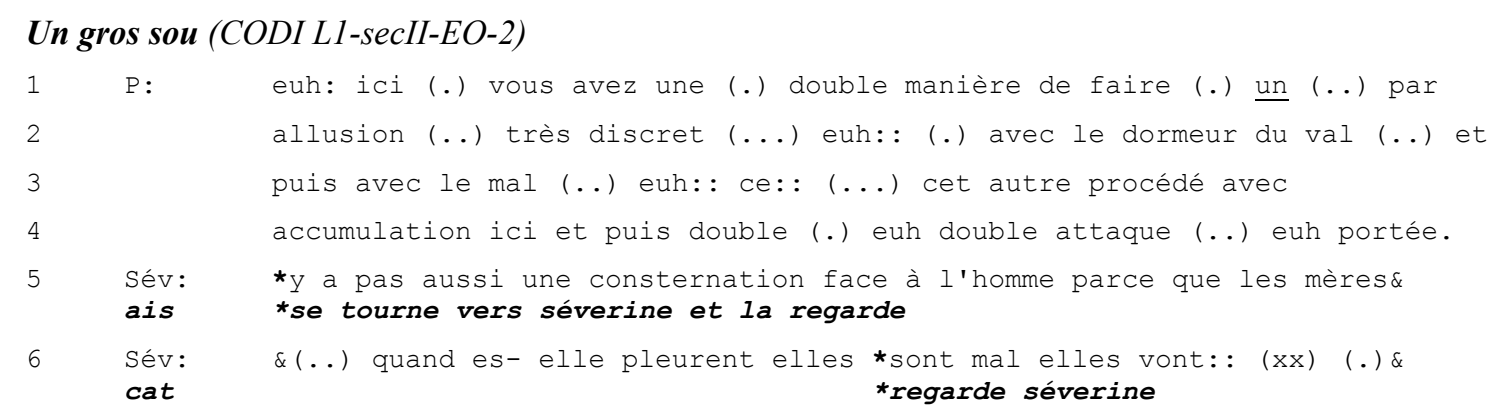


Discours, pragmatique et interaction DOI $10.1051 / \mathrm{cmlf0} 261$

\&et on lui donne un gros sous lié dans leur mouchoir (..) donc même en voyant toute cette (.) cette horreur (..) *autour d'elles (..)\&

\section{*regarde sa feuille}

\&elles vont quand même elles prient dieu (elles vont- elles lui) donnent un gros sou donc en fait euh c'est une consternation aussi face à l'homme (.) face à: (.) à l'homme qui change pas face aux éléments qui sont devant lui?

*(1.5)

* lève les yeux de sa feuille et regarde $P$

mais pourquoi elles lui donnent *un gros sou?

* regarde aishé

*(2.6) ((rires discrets de deux E))

* regarde $P$

*ben c'est comme si tu *le pries tu sais qu'il va rien faire mais\& *regarde ariane

\section{*regarde ariane}

\&tu lui donne *un gros sou (parce que) ça va rien faire\& *regarde à droite dans le vide

\&*mais c'est par con[science]

*regarde aishé

*[mais c'est] peut-être comme les grecs là qui qui\& *regarde aishé

\&mettent des petits sous sur les yeux? pour le:=

* [non mais c'est (xx)]

* [=non (.) non=non c'était pas] ça (.) c'est: c'est (..)\&

*regarde $P$

* regarde catherine

[ (benô̂t) ?]

\&[C'est] pour donner de l'argent à l'église

* $[($ hein) $]$

* $\left[{ }^{\circ} \mathrm{ah}:{ }^{\circ}\right]$

*[pour] que: *(il vienne les aider en fait)

* regarde sa feuille

*regarde séverine

*ah oké

* regarde sa feuille

*(maintenant c'est) * (xxxxxxxxxxxxx) [(xxx)]

* regarde aishé

*regarde catherine

[pourquoi est-ce qu'ils donnent un

gros] *sou. (.) est-ce que ces femmes donnent un gros sou.

*regarde $P$

*regarde $P$

(2.6)

enfin *soit c'est- enfin y a *plusieurs raisons?\&

*regarde séverine

*regarde séverine

\& (1.1) euh dans l'église on- on donne un *gros sou pour a-£

*regarde $P$

*regarde sa feuille

\&*pour donner aux pauvres *(..) ou soit (.)\&

*regarde séverine*

* regarde $P$ 


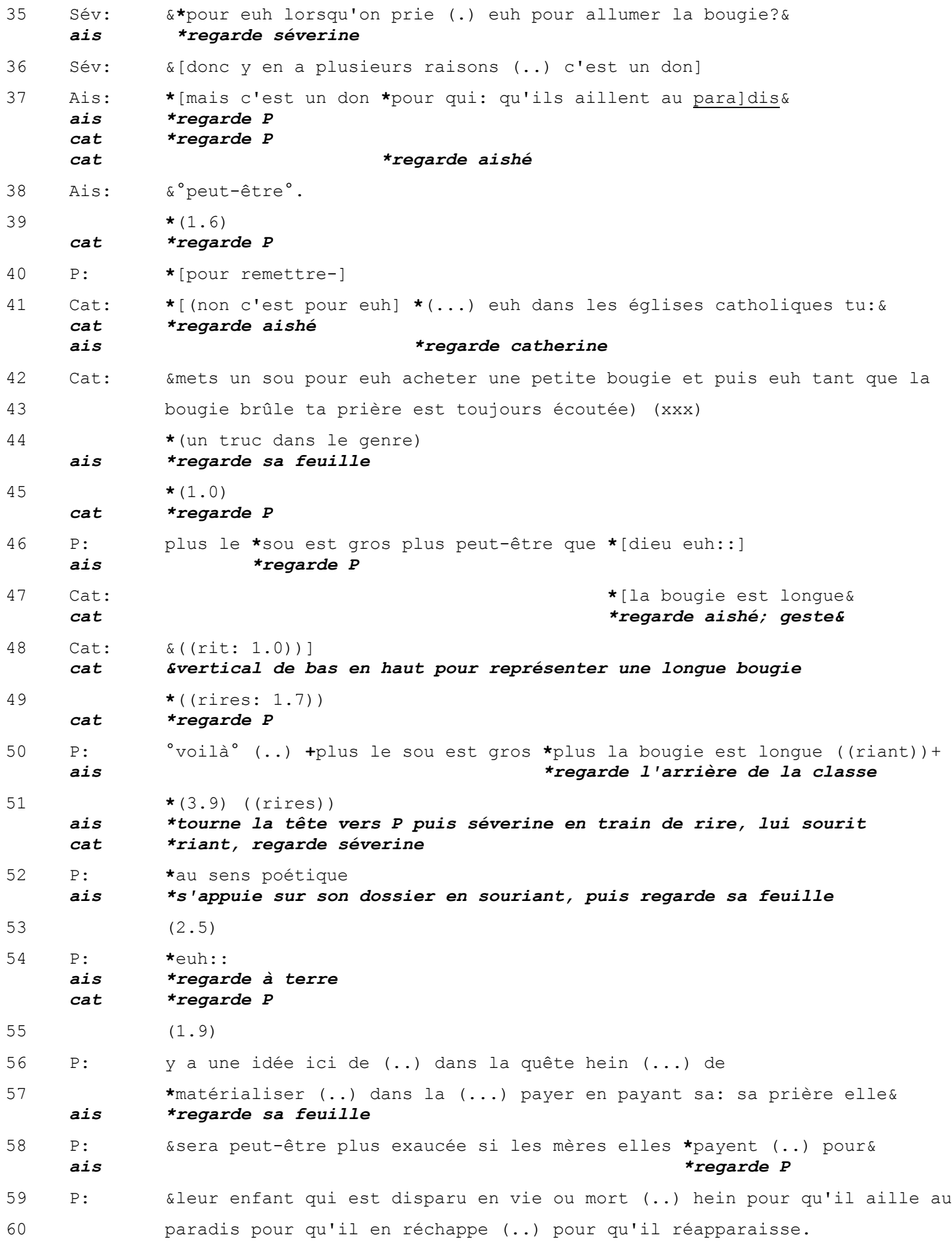

Note: $\mathrm{P}=$ enseignant, Ari $=$ Ariane, Ais $=$ Aishé, Cat $=$ Catherine, Sév $=$ Séverine .

Séverine et l'enseignant sont hors-champ de la caméra.

Cet extrait débute avec un long monologue de l'enseignant (durée: environ une minute; seules les quatre dernière lignes sont transcrites ici), qui contraste deux poèmes de Rimbaud. A la fin de ce monologue, 
une élève, Séverine (hors-champ de la caméra), enchaîne sur l'interprétation de l'enseignant. Ce faisant, elle fait référence au dernier tercet du poème Le Mal, dans lequel il est question de mères donnant à Dieu 'un gros sou lié dans leur mouchoir'. La proposition interprétative de Séverine (1.5-11) est une demande de confirmation présentée comme soumise à l'évaluation de l'enseignant, ce qui s'observe dans le fait que le début de son tour prend la forme d'une question (y a pas aussi, 1.5), reformulée ensuite sur le mode affirmatif, mais qui se termine par une intonation interrogative montante (1.11). Cependant, à la suite du tour de Séverine, l'enseignant ne prend pas la parole. S'ensuit une pause de 1.5 seconde (1.12) qui ouvre de fait un espace pour l'auto-sélection. Aishé prend alors la parole pour demander une explication quant à la signification ou la fonction du gros sou (1.13). A nouveau, bien qu'Aishé s'oriente visiblement vers l'enseignant, ce dernier ne fournit pas l'explication demandée (pause de 2.6 secondes, 1.14). Cette absence répétée de réactions verbales de l'enseignant amène les élèves à répondre eux-mêmes à la sollicitation d'Aishé. Plusieurs élèves prennent alors la parole, et la suite de l'extrait (1.15-46) est donc constituée de l'élaboration collective d'une explication du gros sou.

L'intérêt de cette séquence dans l'optique d'une analyse des cadres de participation émergents réside dans la manière dont les orientations multimodales des participants se modifient de façon localement gérée. Suite à la demande d'explication d'Aishé, nous observons une transition progressive d'un cadre de participation impliquant l'enseignant comme transmetteur de savoir et évaluateur face aux élèves, à un cadre géré par les élèves eux-mêmes. Alors qu'Aishé est orientée vers l'enseignant lorsqu'elle pose sa question, elle se tourne vers Ariane quand celle-ci prend la parole pour initier la séquence d'explication (1.15). Par contre, Ariane reste orientée vers l'enseignant au moment de commencer son explication, bien que celle-ci soit clairement adressée à Aishé (cf. notamment l'usage du pronom $t u, 1.15$ ). C'est seulement dans un second temps qu'Ariane se tourne vers Aishé (1.17). Dès qu'elle croise le regard d'Ariane, Aishé prend la parole pour lui proposer sa propre interprétation de la fonction du gros sou (1.18). La transition d'un cadre enseignant-élève du cadre élève-élève s'effectue dans le changement progressif des orientations corporelles: Aishé, qui regardait l'enseignant, tourne son regard vers Ariane, qui la regarde en retour. Ce contact visuel ratifie le changement de cadre. Le fait que la transition soit accomplie dès le moment où Aishé reprend la parole (1.18) est corroboré par la suite de l'interaction, dans laquelle Benoît et Catherine prennent la parole par auto-sélection sans passer par l'enseignant (1.20-21). Alors que Benoit et Catherine interviennent simultanément, Aishé se tourne vers Catherine (1.21) qui est ainsi ratifiée comme locutrice et continue son explication (1.21-23) jusqu'à obtenir un signal de compréhension de la part d'Aishé (ah, 1.25), alors que Benoît s'interrompt, n'ayant pas bénéficié de son attention. Le fait que l'enseignant sélectionne Benoît en le nommant (1.22) n'y change rien, ce qui montre que la configuration interactionnelle en cours privilégie l'interaction gérée par les élèves eux-mêmes et non par l'enseignant. Ces observations rejoignent ainsi les mécanismes dégagés dans l'analyse du premier exemple: la transition d'un cadre participatif à un autre, ainsi que la gestion de la coexistence de plusieurs cadres en parallèle font l'objet d'un accomplissement conjoint par les participants au moyen de ressources multimodales (lexique, structures grammaticales, regards, postures corporelles).

Après qu'Aishé a manifesté sa compréhension (cf. ah, 1.25 et ah oké, 1.27) de l'explication accomplie conjointement par Ariane (1.15-17), Catherine (1.21-23) et Séverine (1.26), l'enseignant reprend la parole (1.29-30) non pour évaluer l'explication mais pour relancer les élèves en reformulant la question initiale d'Aishé. Il pousse ainsi les élèves à réfléchir non seulement sur la signification du gros sou mais sur les fonctions accomplies par le don d'argent fait à l'église. En posant cette question, l'enseignant d'une part manifeste que pour lui le sujet n'est pas épuisé mais qu'il peut encore être développé. D'autre part, il ne se pose pas en expert évaluateur mais simplement en médiateur d'une discussion qui se joue entre les élèves. Suite à la question de l'enseignant, Séverine (hors-champ de la caméra) propose une explication qui se caractérise par un tour long possèdant une solide structure d'ensemble (projection, enfin y a plusieurs raisons, répétition en fin de tour, donc y a plusieurs raisons, 1.32-36). Durant l'explication de Séverine, Aishé regarde alternativement cette dernière et l'enseignant, visiblement en attente d'une évaluation de la part de l'enseignant - puisque dans la salle de classe, les contributions des élèves peuvent potentiellement faire l'objet d'une évaluation. Ce faisant, elle se positionne comme auditrice ratifiée (Goffman, 1981) personne observant l'échange entre l'élève et l'enseignant. Toutefois, le fait qu'elle regarde ces deux interactants ne consiste pas seulement en un suivi de la circulation de la parole. Bien plus, l'explication 
proposée par Séverine revêt pour Aishé un enjeu particulier, dans la mesure où c'est elle qui a déclenché cette séquence d'explication par sa question initiale (1.13), qui a ensuite été reformulée par l'enseignant (1.29-30). Ainsi, par son regard attentif, elle se positionne également comme destinataire de l'explication. Dès qu'elle en a l'opportunité, Aishé propose son interprétation et se repositionne de la sorte comme participante centrale de la discussion. Elle saisit la première occasion pour prendre la parole en s'autosélectionnant à un point de complétude potentiel sur le plan syntaxique, sémantique et pragmatique (Sacks, Schegloff \& Jefferson, 1974) (1.37). Le chevauchement (1.36-37) résulte du développement de Séverine au-delà de ce point de complétude. Lorsqu'Aishé soumet son interprétation, elle regarde l'enseignant uniquement, comme lors de sa question initiale (1.37, cf. 1.13), le sélectionnant implicitement comme destinataire principal de son propos.

Comme dans la situation initiale, l'enseignant ne réagit pas immédiatement de manière verbale (cf. pause de 1.6 seconde, 1.39). L'enseignant ne prenant pas la parole, les élèves disposent donc d'un espace pour s'auto-sélectionner. C'est ce que fait Catherine: après avoir regardé l'enseignant pendant toute la durée de la pause (1.39), une fois qu'elle s'est assurée de l'absence de réaction immédiate de sa part, elle se tourne vers Aishé et prend la parole pour réfuter la proposition d'Aishé (1.41-44). L'enseignant quant à lui, qui s'est mis à parler exactement en même temps que Catherine (1.40), s'interrompt et laisse l'élève terminer son tour, abandonnant ainsi son projet communicatif. C'est seulement lorsque Catherine a terminé son tour (1.44) et se retourne vers lui, et après une nouvelle pause de 1.0 seconde (1.45), que l'enseignant reprend la parole (1.46). Durant toute la séquence d'explication collective, l'enseignant se met en retrait sur le plan verbal, ne répondant pas aux sollicitations des élèves ou leur laissant la priorité, les invitant ainsi à gérer eux-mêmes le développement d'un cadre de participation entre pairs. De par son comportement, il crée et maintient ainsi l'espace nécessaire à l'élaboration collective de l'explication, ce qui montre la manière dont les configurations interactionnelles sont négociées par l'ajustement mutuel des participants sur le plan local et ne sont pas prédéterminées à l'avance.

Dans la dernière partie de l'extrait, la présence de nombreux rires (Catherine 1.48; enseignant, 1.50, collectif, 1.49 et 51$)$ ainsi que l'augmentation des pauses $(1.51 ; 1.53 ; 1.55)$ ralentissent la dynamique interactionnelle, ce qui peut indiquer un mouvement vers une clôture de l'épisode conversationnel. La séquence d'explication se clôt sur une conclusion co-énoncée entre l'enseignant et Catherine. La structure syntaxique bipartite amorcée par l'enseignant (1.46) et complétée par Catherine (1.47) sous forme de complétion anticipée tardive (cf. Lerner, 1996) s'apparente à un dicton populaire (plus le sou est gros plus la bougie est longue, 1.46-50) qui fonctionne comme 'le mot de la fin'. Les rires partagés de l'enseignant et des élèves témoignent de l'aboutissement réussi de l'explication, i.e. le partage des connaissances qui renforce la complicité entre les membres du groupe. D'autre part, la co-énonciation et les rires communs permettent de réunir l'enseignant et les élèves dans une participation conjointe à l'interaction et ainsi d'assurer la transition entre la fin de l'explication, coïncidant avec la clôture du cadre de participation entre pairs, et la reprise de l'analyse de texte par l'enseignant (1.56 et suivantes). Aishé baisse les yeux en direction du sol (1.54): elle se retire du cadre de participation, qui se dissout en raison de l'absence de regards mutuels. Ce faisant, elle abandonne visiblement le rôle actif d'interlocutrice qu'elle endossait depuis le début de la séquence, tandis que l'enseignant retourne à l'activité initiale de commentaire de texte littéraire.

Cet extrait ainsi que le précédent montrent que les transitions entre cadres de participation et la création de nouveaux cadres pour accomplir une activité entre pairs donnent aux élèves l'opportunité de prendre en charge eux-mêmes la gestion de l'interaction, de l'ouverture à la clôture de la séquence, ce qu'ils font rarement. D'autre part, elles sont aussi un lieu privilégié pour construire de nouvelles connaissances de manière négociée et collaborative.

\section{Conclusion}

Nos analyses ont montré que l'analyse conversationnelle multimodale d'interactions en classe offre un apport essentiel à la compréhension du déroulement de ces interactions, en permettant une description détaillée de la logique localement négociée des échanges. Une telle approche permet ainsi de rendre 
compte de la dynamique communicative des interactions dans toute leur complexité, et notamment des transitions progressives entre cadres de participation ou de la coexistence de plusieurs cadres. Dans un second temps, ces analyses permettent de réfléchir aux potentiels acquisitionnels mis en œuvre dans les interactions en classe de français langue première, dont les objectifs pédagogiques concernent le développement des compétences langagières en articulation avec l'acquistion de contenus disciplinaires (portant principalement sur la littérature). Or, si les interactions formatées par la structure tripartite IRF permettent la transmission et la vérification des connaissances de et par l'enseignant, elles semblent moins propices au développement de la compétence d'interaction des élèves, dont la marge de manœuvre est fortement limitée. Par contre, lorsque la séquence IRF est abandonnée au profit d'une configuration interactionnelle favorisant les échanges entre pairs, les élèves ont l'occasion de prendre en charge euxmêmes la gestion de l'interaction. Cette prise en charge engendre une participation dynamique de leur part, qui s'observe tant sur le plan linguistique (tours plus longs et mieux argumentés, cf. Pekarek, 1999) que multimodal, dimension que nous avons privilégiée dans nos analyses: les élèves 'suivent' des yeux la circulation de la parole et cherchent à trouver des correspondances dans leur texte, marquant par là leur intérêt et leur concentration. D'autre part, une telle configuration interactionnelle permet la construction négociée des connaissances (l'interprétation d'un poème, la signification d'une expression) en collaboration par les élèves eux-mêmes, dans des échanges intrinsèquement authentiques (van Lier, 1988; Springer, 2002) qui font sens pour eux en ce qu'ils prennent activement en charge leurs propres apprentissages en fonction de leurs besoin, ce qui renforce également leur motivation.

Les séquences que nous avons analysées se caractérisent par leur richesse interactionnelle, leur potentiel acquisitionnel mais aussi leur rareté. Nous pensons que nos analyses peuvent avoir un impact sur les pratiques éducatives, et souhaitons soumettre à réflexion nos résultats, concernant en particulier le rôle de l'enseignant qui est capital dans les deux extraits que nous avons analysés: l'enseignant ne se met pas simplement en retrait de l'interaction, il manifeste cette mise en retrait qui de ce fait, loin de déstabiliser les apprenants, les encourage à participer davantage. D'autre part, il ne sanctionne pas les discussions 'semi-officielles' entre élèves qui chuchotent entre eux, ce qui a pour conséquence que de telles discussions peuvent acquérir un statut officiel et venir enrichir le cours principal de l'interaction. Ainsi, le comportement de l'enseignant, loin de n'être qu'une forme de passivité ou d'effacement, est un processus complexe qui demande une certaine expertise pouvant faire l'objet d'une sensibilisation lors de la formation d'enseignant. La valorisation de cette expertise et la pratique en vue de son développement chez les enseignants en formation permettrait ainsi d'augmenter la fréquence de telles configurations interactionnelles en classe.

D'autre part, nous souhaitons étendre nos analyses multimodales également aux élèves prenant rarement la parole en classe. Nous pensons que l'observation multimodale permet de rendre compte de la 'densité' de participation des élèves, notamment de leur degré d'écoute et d'implication. De tels outils analytiques pourraient donc s'avérer fructueux pour mieux décrire le comportement et dégager les opportunités d'apprentissage des élèves restant silencieux, qui sont trop rarement pris en compte dans les études sur les interactions en classe.

\section{Références}

Cicurel, F. (2002). La classe de langue. Un lieu ordinaire, une interaction complexe. AILE, 16, 145-163.

Coughlan, P. \& Duff, P. (1994). Same task, different activities: Analysis of a SLA task from an activity theory perspective. In J. P. Lantolf \& G. Appel (éds.), Vygotskian approaches to second language research. Norwood/NJ: Ablex, 173-193.

Dabène, L. (1984). Pour une taxinomie des opérations métacommunicatives en classe de langue étrangère. ELA 55, $39-46$.

Fasel Lauzon, V. \& Berger, E. (2008). Collective talk in multi-party interactions. Communication orale, AAAL Annual Conference, Washington D.C. (USA), 29 mars-1 avril 2008.

Gajo, L. \& Mondada L. (2001). Acquisition et interaction en contexte. Fribourg: Editions Universitaires. 
Goffman, E. (1981). La Position. In E. Goffman (ed.), Façons de parler. Paris: Editions de Minuit, 133-166.

Goodwin, Ch. (1979). The interactive construction of a sentence in natural conversation. In G. Psathas (ed.), Everyday language: Studies in ethnomethodology. New York: Academic Press, 91-121.

Goodwin, Ch. (2000). Action and embodiment within situated human interaction. Journal of Pragmatics, 32, 14891522 .

Goodwin, Ch. (2007a). Participation, Stance, and Affect in the Organization of Activities. Discourse and Society, 18/1, 53-73.

Goodwin, Ch. (2007b). Interactive Footing. In E. Holt \& R. Clift (éds.), Reporting Talk: Reported Speech in Interaction. Cambridge: University Press, 16-46.

Goodwin, Ch. \& Goodwin, M. H. (2004). Participation. In A. Duranti (éd), A companion to Linguistic Anthropology. Oxford: Blackwell, 222-244.

Gülich, E. \& Mondada, L. (2001). Konversationsanalyse. In G. Holtus, M. Metzeltin \& Ch. Schmitt (éds.), Lexikon der Romanisitischen Linguistik. Tübingen: Niemeyer, 196-250.

Hall, J.K. (2004). Language and learning as an interactional achievement. The Modern Language Journal, 88 (4), 606-612.

Hall, J.K. (2007). Redressing the roles of correction and repair in research on second and foreign language learning. The Modern Language Journal, 91 (4), 510-525.

Heritage, J. (2004). Conversation Analysis and Institutional Talk. In K. L. Fitch \& R. E. Sanders (éds.), Handbook of language and social interaction. Mahwah: Erlbaum, 103-148.

Heritage, J. \& Greatbach, D. (1991). On the institutionnal character of institutional talk: the case of news interviews. In D. Boden \& D. Zimmerman (éds.). Talk and social structure. Oxford: Blackwell, 93-137.

Hymes, D. (1974). Foundations of Sociolinguistics: An Ethnographic Approach. Philadelphia: University of Pennsylvania.

Lerner, G. (1993). Collectivities in action: establishing the relevance of conjoined participation in conversation. Text, $13,213-245$.

Lerner, G. (1996). On the 'semi-permeable' character of grammatical units in conversation: conditional entry into the turn space of another speaker. In E: Ochs, E. A. Schegloff \& S. Thompson (éds.). Interaction and Grammar. Cambridge: Cambridge University Press, 238-276.

Lave, J. \& Wenger, E. (1991). Situated learning: legitimate peripheral participation. Cambridge: Cambridge University Press.

Lier (van), L. (1988). The Classroom and the Language Learner. London: Longman.

Markee, N. (2004). Zones of Interactional Transision in ESL Classes. The Modern Language Journal, 88 (4), 583596.

Mehan, H. (1979). Learning Lessons. Social Organization in the Classroom. Cambridge (MA): Harvard University Press.

Mehan, H. (1985). The Structure of Classroom Discourse. In T. A. van Dijk (éd.), Handbook of Discourse Analysis vol.3. London: Academic Press, 119-131.

Mondada, L. (2003). Observer les activités de la classe dans leur diversité: choix méthodologiques et enjeux théoriques. In J. Perera, L. Nussbaum \& M. Milian (éds.), L'Educacio lingüistica en situacions multiculturals $i$ multilingües. Barcelone: ICE Universitat de Barcelona, 49-70.

Mondada, L. \& Pekarek-Doehler, S. (2004). SLA as situated practice: Task accomplishment in the French Second Language Classroom. The Modern Language Journal, 88 (4), 501-518.

Mortensen, K. (à par.). Establishing recipiency in pre-beginning position in the second language classroom. Discourse processes.

Pekarek, S. (1999). Leçons de conversation. Fribourg: Editions Universitaires. 
Pekarek Doehler, S. (2002). Formes d'interaction et complexité des tâches discursives dans des activités conversationnelles en classe de L2. In F. Cicurel \& D. Véronique (éds.), Discours, action et appropriation des langues. Paris: Pubications de la Sorbonne Nouvelle, 117-130.

Pomerantz, A. (1984). Agreeing and disagreeing with assessments: some features of preferred/dispreferred turn shapes. In J. M. Atkinson \& J. Heritage (eds.), Structures of social action: studies in Conversation Analysis. Cambridge: Cambridge University Press, 55-101.

Sacks, H. (1992). Lectures on conversation. Oxford: Blackwell.

Sacks, H., Schegloff, E. A. \& Jefferson, G. (1974). A Simplest Systematics for the Organization of Turn-Taking for Conversation. Language 50 (4), 696-735.

Schegloff, E. A. (1988). Discourse as an Interactional Achievement II: An Exercicse in Conversation Analysis. In D. Tannen (éd.), Linguistics in Context: Connecting Observation and Understanding. Lectures form the 1985 LSA/TESOL and NEH Institutes. Norwood: Ablex, 135-158.

Schegloff, E. A. (1995). Discourse as an interactional achievement III: The omnirelevance of action. Research on language and social interaction, 28 (3), 185-211.

Schegloff, E. A. \& Sacks, H. (1973). Opening up closings. Semiotica, 8 (3), 289-327.

Seedhouse, P. (2004). The Interactional Architecture of the Language Classroom: A Conversation Analytic Perspective. Oxford: Blackwell.

Sfard, A. (1998). On two metaphors for learning and the dangers of choosing just one. Educational Researcher, 27(2), 4-13

Sinclair, J. McH. \& Coulthard, R. M. (1975). Towards an Analysis of Discourse. The English used by teachers and pupils. London: Oxford University Press.

Springer, C. (2002). Recherches sur l'évaluation en L2: de quelques avatars de la notion de 'compétence'. Notions en Question 6, 61-73.

Wells, G. (1993). Reevaluating the IRF Sequence: A Proposal for the Articulation of Theories of Activity and Discourse for the Analysis of Teaching and Learning in the Classroom. Linguistics and Education 5, 1-37.

\section{Annexe: conventions de transcription}

\begin{tabular}{|l|l|}
\hline$(\cdot)$ & petites pauses (max 0.3 secondes) \\
\hline$(\ldots)$ & pauses moyennes (max 0.6 secondes) \\
\hline$(\ldots)$ & longues pauses (max 1.0 seconde) \\
\hline$(1.2)$ & pauses de plus de 1 seconde \\
\hline$: \quad: \quad:::$ & allongements de syllabe \\
\hline$[\quad]$ & début et fin de chevauchement de parole \\
\hline$($ bonjour) & transcription incertaine \\
\hline$?$ & intonation montante \\
\hline$\cdot$ & intonation descendante \\
\hline- & troncation / interruption \\
\hline$=$ & enchainement rapide \\
\hline$\&$ & continuation du tour de parole \\
\hline très bien & emphase/accentuation \\
\hline
\end{tabular}




\begin{tabular}{|l|l|}
\hline${ }^{\circ}$ doucement & \\
\hline$(($ rit: 1.0$))$ & volume faible \\
\hline+ bla $($ riant $)++$ & commentaire du transcripteur \\
\hline * & début d'un mouvement, d'un changement de position ou de regard \\
\hline
\end{tabular}

${ }^{1}$ En effet, la notion de participation s'est établie comme un concept analytique pertinent dans le cadre d'analyses dans le domaine institutionnel-éducatif (cf. Goodwin \& Goodwin, 2004, à propos de Mehan, 1979) aussi bien que le domaine de l'apprentissage (cf. Sfard, 1998 pour une discussion de deux métaphores dominantes en vigueur dans le domaine des recherches sur l'apprentissage: acquisition et participation; cf. Lave \& Wenger, 1991 pour la notion de participation périphérique légitime).

${ }^{2}$ Il convient de noter que déjà dans le modèle SPEAKING de Hymes (1974), les différents types de participants - le P dans SPEAKING - et leurs rôles respectifs occupaient une place importante.

3 Traduction: ... comment les interlocuteurs en tant qu'acteurs incarnés et l'organisation de la parole sont intégrés dans un cours d'action commun.

${ }^{4}$ Traduction: En particulier, cette approche implique de se focaliser sur la manière dont les règles et les pratiques interactionnelles sont sans cesse mises en œuvre par les participants dans la construction de la compréhension mutuelle de la place où ils se trouvent au sein de l'interaction. La dimension réflexive de l'action sociale est centrale dans ce processus: par leurs actions, les participants manifestent une analyse ou leur compréhension de l'événement interactif dans lequel ils sont engagés, mais en agissant, ils réalisent également une contribution interactionnelle qui fait avancer l'événement sur la base de cette analyse.

${ }^{5}$ Pour des raisons d'espace, nous avons choisi de ne pas intégrer d'images dans cet article. Néanmoins, la transparence de la transcription devra permettre selon nous de rendre compte des détails multimodaux pertinents pour l'analyse. 